\title{
STEREO VISION BASED HYBRID MANUFACTURING PROCESS FOR PRECISION METAL PARTS
}

\author{
Zhiyuan Wang, Renwei Liu, Todd Sparks, Heng Liu, Frank Liou \\ Mechanical and Aerospace Engineering \\ Missouri University of Science and Technology \\ Rolla, Missouri, USA
}

\begin{abstract}
This paper presents the research and development of an automatic hybrid manufacturing process which based on stereo vision and laser scanning technology to produce fully dense metal parts with CNC level precision. High performance metals, such as titanium alloys, nickel superalloys, tool steel, stainless steels, etc., can benefit from this process. Coupling the additive and the subtractive processes into a single workstation, the hybrid process, can produce metal parts with accuracy. The surface quality of the final product is similar to the industrial milling capability. It will certainly impact the future rapid manufacturing industry. To achieve such a system, issues, including the modeling of the metal deposition process, the automated path planning and accurate surface scanning of the hybrid manufacturing process, are summarized.

KEYWORDS: Hybrid manufacturing, Deposition process molding, Stereo Vision, Laser Surface Scanning 1 INTRODUCTION

The Directed Energy Deposition (DED) process referred here is a metal additive manufacturing process in which metal is added to the part or product, layer by layer, to rapidly manufacture or form the part or product to a predetermined shape. It is a technique that can produce fully dense functional metal parts or tools directly from a CAD system and eliminate the need for intermediate steps. An example of DED process discussed in this paper is shown in Figure 1.
\end{abstract}




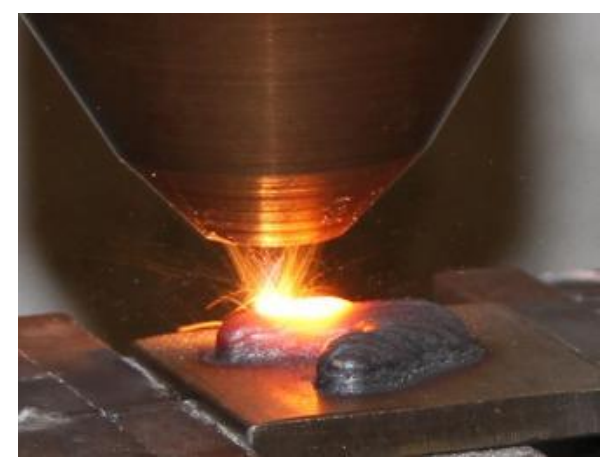

Figure 1. A blown powder metal deposition process is depositing a steel part.

A DED process is especially beneficial for high performance metals, such as fully dense titanium alloys, Inconel, and tool steel, which are difficult for traditional CNC machines or rapid prototyping (RP) machines to fabricate. For example, titanium and its alloys have proven to be technically superior and cost-effective materials for a wide variety of aerospace, industrial, marine, medical, and commercial applications. Parts or products cast and/or machined from these high performance metals are very expensive, partly due to the processing difficulties and complexities during machining and casting. DED processes however have been found to be very cost effective because they can produce near-net shape parts from these high performance metals with little or no machining. However, as DED processes cannot build support materials, multi-axis capability is critical in metal deposition technologies. The hybrid manufacturing process here combines laser deposition and machining processes to develop a rapid manufacturing process to build functional metal parts. This paper summarizes the research and development of such a hybrid process, including modeling and understanding of the direct laser deposition process for distortion, and automated process planning of the hybrid process.

\section{DEPOSITION PROCESS MODELING}

The basis of DED process is a sound microstructure which is dominated by the created melt pool during deposition. Melt pool formed during laser deposition is a critical factor and melt pool geometry is a crucial factor in determining deposition quality. To optimize process parameters, a deep understanding of the underlying mechanisms is beneficial. A mathematical model, as shown in Figure 2, was developed to simulate the coaxial laser cladding process with powder injection, which includes laser-substrate, laserpowder and powder-substrate interactions [1]. The model considers most of the associated phenomena, such as melting, solidification, evaporation, evolution of the free surface and powder injection. The fluid 
flow in the melt pool, which is mainly driven by Marangoni shear stress as well as particle impinging, together with the energy balances at the liquid-vapor and the solid-liquid interfaces are investigated. Powder heating and laser power attenuation due to the powder cloud are incorporated into the model in the calculation of the temperature distribution. The influences of the powder injection on the melt pool shape, penetration, and flow pattern are predicted by comparison between cases with powder injection and without powder injection. Dynamic behavior of the melt pool and the formation of the clad are simulated. The effects of the process parameters on the melt pool dimension and peak temperature are further investigated based on the validated model.

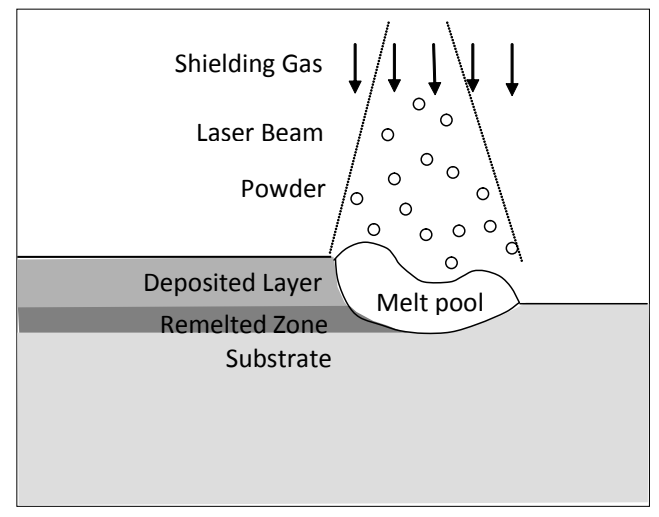

Figure 2. Schematic diagram of the calculation domain for laser deposition process.

\section{DISTORTION ANALYSIS}

Highly localized heating and cooling during DMD process produces nonuniform thermal ex- pansion and contraction, resulting in complicated distribution of residual stresses in the heat affect zone and unexpected distortion in the whole structures. The residual stresses may promote fracture and fatigue and induce unpredictable buckling during the service of deposited parts and the distortion is often detrimental to the dimensional accuracies of structures. Therefore, it is vital to predict the behavior of materials after DMD process and optimize the design/manufacturing parameters to control the residual stresses and distortion.

During DED process, the substrate will continuously experience expansion and shrinkage and finally keep a deformed shape. Deformation in y direction, shown in Figure 3 , is the main deformation under consideration and is observed by both experiments and simulations shown in Figure 4. 


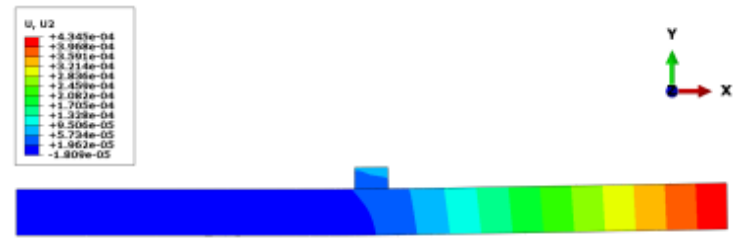

Figure 3: Deformation of substrate in y direction

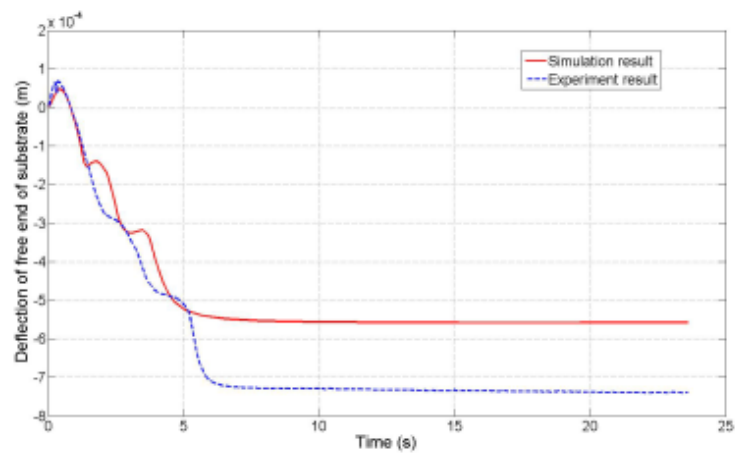

Figure 4: Simulation and experiment results of deflection of substrate.

The distortion analysis tool allows the planning of an effective hybrid manufacturing process so that the distortion effect could be minimized through the machining process.

\section{HYBRID MANUFACTURING SYSTEM}

In order to expand the applications of DED processes, multi-axis capability is needed. A multi-axis rapid manufacturing system can be hardware-wise configured by adding extra degrees of mobility to a deposition system or by mounting a laser deposition device on a multi-axis robot. The configuration could also be a hybrid system in which a laser deposition system is mounted on a multi-axis CNC machine. With the addition of extra rotations, the support structures may not be necessary for the deposition process in order to build a complicated shape. Due to the nature of the deposition process, it is driven by a so-called "slicing" procedure, which uses a set of parallel planes to cut the object to obtain a series of slicing layers. So far, the slicing software on the market is only able to handle $2.5 \mathrm{D}$ slicing in which the building/slicing direction is kept unchanged and it lacks the capability of changing directions to fully explore the capability of multiple degrees of freedom.

This process uses laser deposition for material deposition and CNC milling for material removal. As shown in Figure 5, it includes two major systems: a laser deposition system and a CNC milling machine system. 


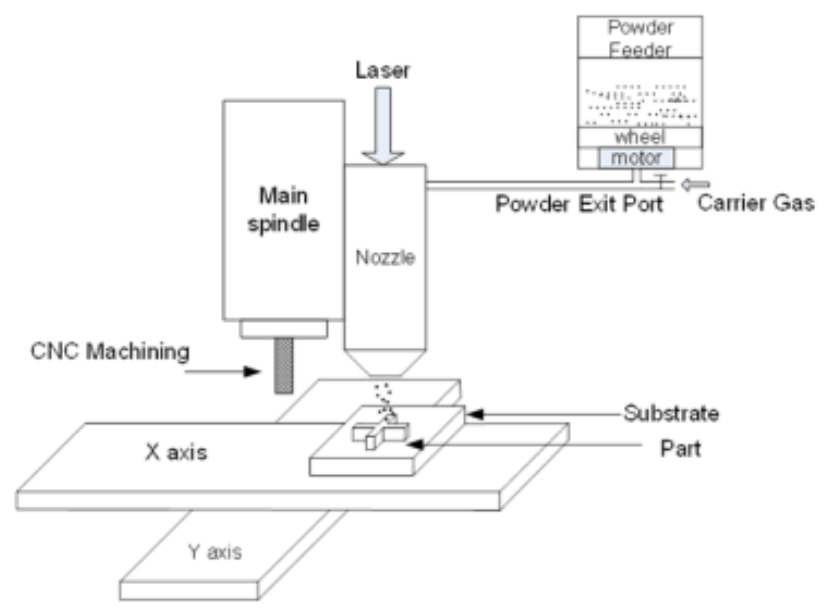

Figure 5. A hybrid manufacturing system: laser deposition for material deposition and CNC milling for material removal.

The laser deposition system and CNC milling machine work in shifts in a five-axis motion mode. The laser deposition system consists of a laser and a powder feeder. In a conventional 2.5-D laser deposition process to create three-dimensional parts, overhang and top surfaces of hollow parts must be supported. Often support materials for functional metal parts are not feasible. Moreover, it increases the build time of the part and necessitates a time-consuming post-processing. Additionally use of support increases the build time of the part and necessitates a time-consuming post-processing. With a five-axis deposition process integrated with five-axis machining, these obstacles can be removed.

Measurement with high resolution is the basis of precise manufacturing [2-3]. Measuring with camera could get numerous information in a short time but not accurate, the laser sensor is precision enough but relatively slow. Stereo vision camera couple with laser displacement sensor archive a balance between speed and precision for automation path planning [4]. As shown in Fig. 6, two level of measuring will be taken in this process: Rough and Precise. Two-eye camera adopted with stereo vision algorithm could provide the spatial information for this hybrid manufacturing system, including the position, size, shape of part, substrate, machine tool and the deposition nozzle. The work of precisely scanning in specific working area will finished by Laser displacement sensor, which the resolution of point clouds could reach to 10 micron. 


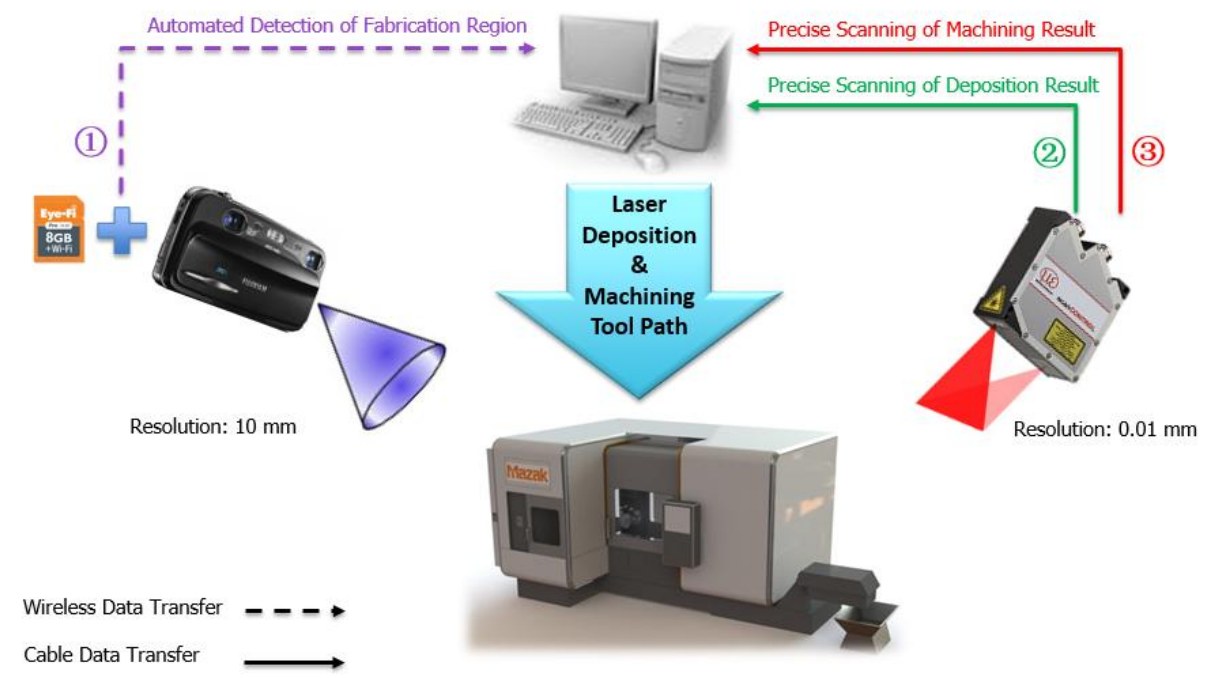

Figure 6. Stereo vision based path planning for hybrid manufacturing

\section{STEREO VISION BASED HYBRID PROCESS PLANNING}

The major issue to make an effective hybrid process is the automation of the system. Process planning, simulation, and tool path generation of a hybrid manufacturing process allow the designer to visualize and perform the part fabrication from the desktop. The Laser Aided Manufacturing Process Planning system uses B-Rep models as input and generates a description that specifies contents and sequences of operations. The objective of the process planning is to integrate the five-axis motion and depositionmachining hybrid processes. The results consist of the subpart information and the build/machining sequence4. Basic planning steps involve determining the base face, extracting the skeleton, decomposing a part into subparts, determining build sequence and direction for subparts, checking the feasibility of the build sequence and direction for the machining process, and optimization of the deposition and machining [5-7].

1) Skeleton Computation

An algorithm for computing the skeleton of the 3-D polyhedron is needed. The algorithm is based on a classification scheme for points on the skeleton computation in which the continuous representation of the medial axis is generated with associated radius functions. Because it is used as a geometric abstraction, the skeleton is trimmed from the facets that touch the boundary of the object along every boundary edge for which the interior wedge angle is less than $\pi$ rad.

2) Part Orientation 
The determination of the base face from which the building process of the part starts is very important. The base face functions as the fixture in the machining process. Therefore, when in the machining process, it must provide resistance against the cutting force. The maximal resistance force depends on the area of the base face.

\section{3) Part Decomposition and Building Direction}

The objective of part decomposition is to divide the part into a set of subparts, which can be deposited and machined. The topology of the part can be obtained from the skeleton. Each branch of the skeleton corresponds to a subpart. One of the partitions that is performed is along a non-planar surface. Therefore, close to the partition area, 3-D layers are needed to build the connection between two subparts. The build direction of a subpart may not be constant. It changes when the part is built layer by layer so that for two adjacent layers, the later layer can be deposited based on the early layer without any support structures. To achieve the non-support build, the build directions need to be along the skeleton.

\section{4) Building Sequence}

The results of decomposition are recorded in an adjacency graph where nodes represent subparts, and edges represent the adjacency relationship between connected nodes. After considering part building order, a directed graph that represents the precedence relationship among subparts can be constructed. From the precedence graph, one can identify in what order the subparts can be built. With the precedence graph, a set of alternative building plans can be generated. Each plan represents a possible building sequence on the decomposed geometry and can be chosen optimally depending upon machine availability or other criteria such as minimum building time.

5) Machinability Check

The main purpose of the machinability check is to choose an optimal building sequence from the sequence set. Local and global collision checks are operated first to choose acceptable sequences since the building direction is different in each sequence. If any kind of collision happens or an undercut plane appears, the corresponding sequence will be discarded. For the rest of the building sequences in the set,

the building ability check and machining time computation is performed to find an optimal building sequence. 
Stereo computer vision technology is adopted to obtain the spatial information of the part and hybrid manufacturing system. Firstly, several markers are put around the target working area on the part for image processing. After the part securely fixed on the CNC, stereo camera will take several stereo images. Then the image processing is done by stereo marker matching to get the value of three dimension coordinates in the camera coordinates system. The stereo camera calibration provide the camera intrinsic and extrinsic parameters. The spatial information in camera coordinate system could be transformed to the hybrid manufacturing environment with these information. This process is to build the relationship between CNC machine and camera coordinate system. And then the $3 \mathrm{~d}$ coordinates of defect area on the Ti64 part in camera coordinate system is transformed to CNC machine. Eq. (1) and Eq. (2) show the method to get the transformation matrix $T_{c a m}^{c n c}$. Four spatial positions which are not in one plane on CNC machine are used to calculate $T_{c a m}^{c n c}$. Read each coordinate of those four positions in CNC machine and then calculate the corresponding coordinate of those four positions in camera coordinates system by stereo computer vision method.

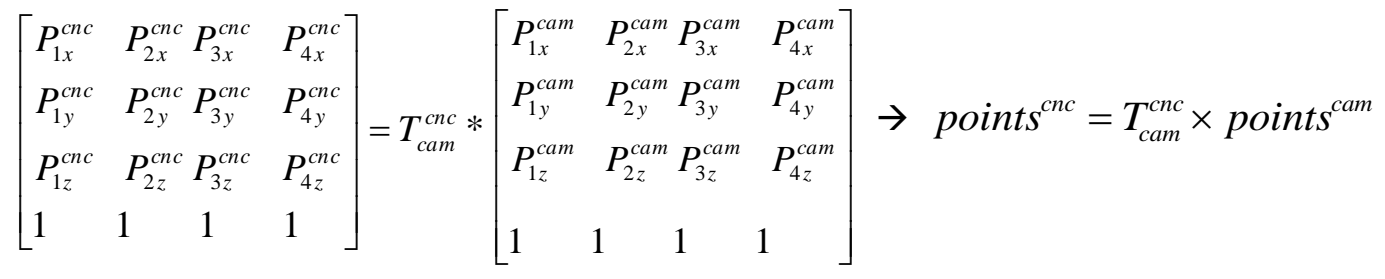

$$
\begin{aligned}
& T_{\text {cam }}^{c n c}=\text { points }^{c n c} \times \operatorname{inv}\left(\text { points }^{\text {cam }}\right)
\end{aligned}
$$

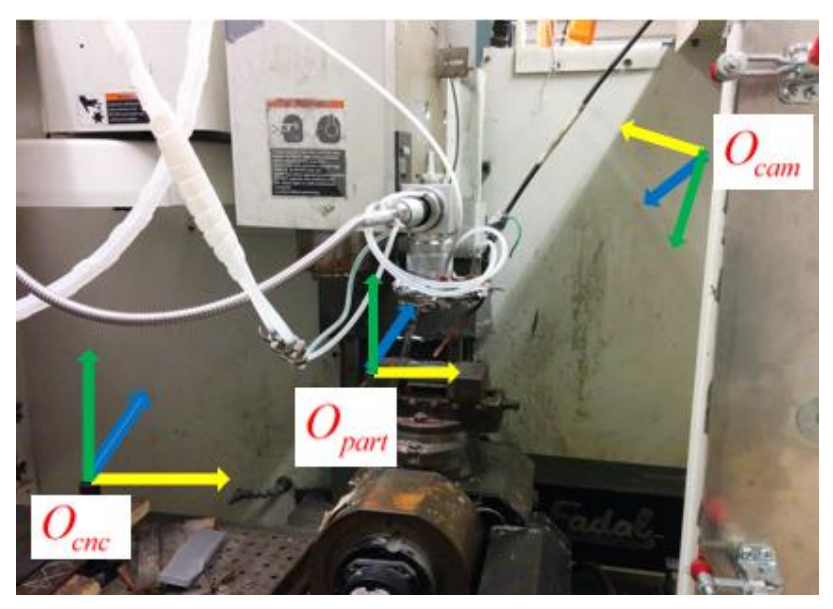

Figure 7. Coordinate Relationship between camera, CNC, and part 


\section{RESULTS}

Laser displacement sensor is used to scan the geometry of deposition result and machining area. The resolution of measurement can be reach to 10 micron. These accurate dimension data is basis for path planning of near-net manufacturing. Point clouds of the deposition area is used to generate the machining tool path. Fig.8 shows the laser displacement sensor is scanning a deposition sample on Ti64 substrate. Fig.9 describes the machining tool path according to this point clouds information.
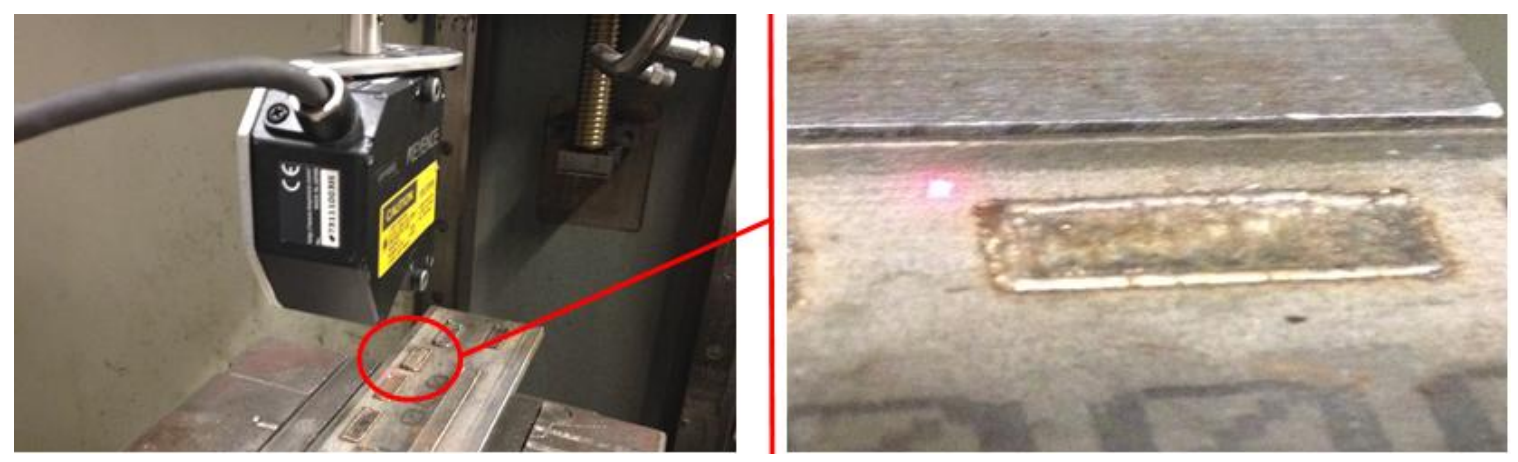

Figure 8. Accurate surface scanning with laser displacement sensor
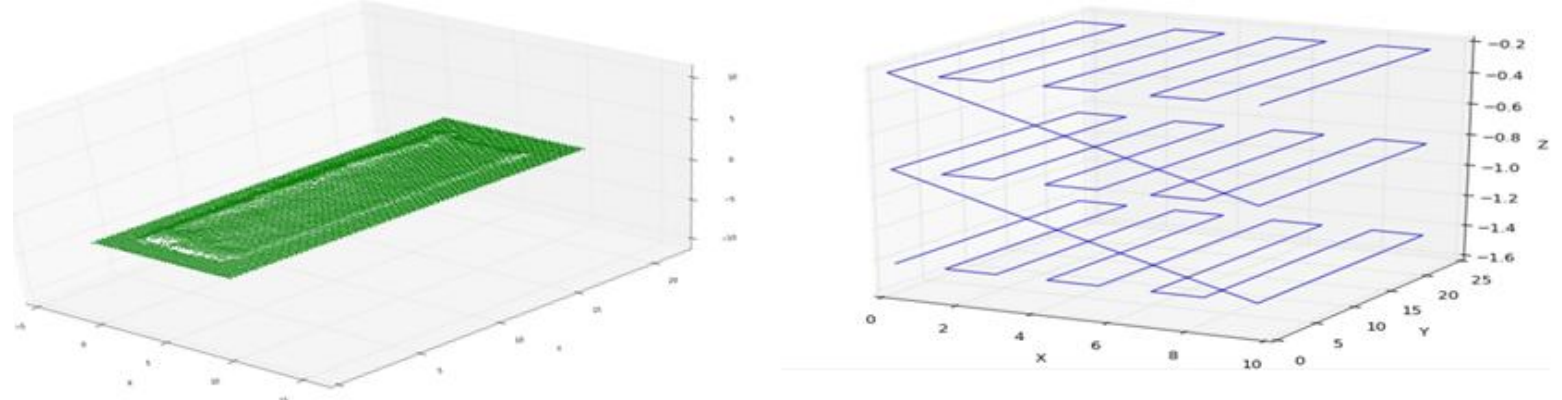

Figure 9. Point clouds of scanning results and the machining tool path

The bearing seat example as shown in Figure 10 (a) and its deposition result are shown here. Figure 10 (b) shows the planed tool path for both deposition and machining, Figure 10 (c) shows the part in $\mathrm{H} 13$ tool steel being deposited, and Figure 10 (d) shows the part after machining. 


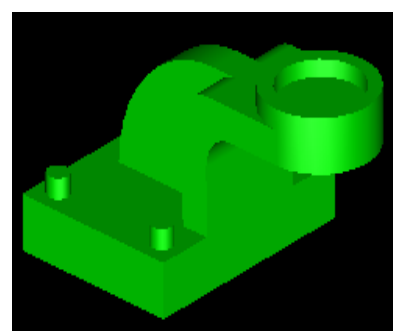

(a)

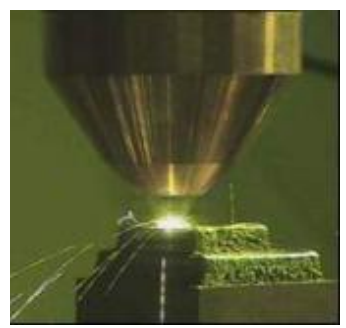

(c)

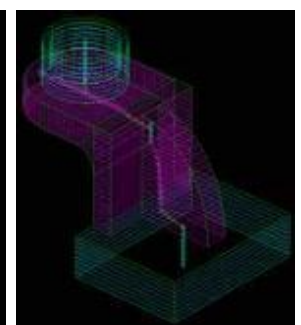

(b)

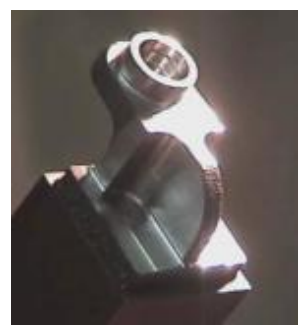

(d)

Figure 10. Hybrid manufacturing of an $\mathrm{H} 13$ tool steel bearing seat part: (a) CAD model (b) Automated generated tool path for deposition and machining; (c) Laser deposition; (d) After machining.

\section{CONCLUSION}

Making precision metal parts is a critical additive manufacturing technology as it can directly produce finished products or parts for high performance applications. The current limitations of additive processes include surface finish, repeatability, and material properties. The research and applications of a stereo vision based hybrid metal manufacturing system are summarized in this paper. The issues include understanding the DED process, the stereo vison aided automated process planning, and laser displacement sensor surface scanning. The modeling and simulation of material-laser interaction help design and set the process parameters for metal deposition. The stereo vison based path planning and laser sensor scanning make the process has less involve with manual operation, enhance the automation and accuracy of additive manufacturing process. The overall goal for process planning is not only to find a solution to build a part but also to look for an answer to produce it in the least amount of time; therefore, the least amount of switching between the machining process and deposition process, the better since each switch requires retreating and relocating the deposition nozzle as well as the machining tool, which may cost extra time. With integration of multi-axis deposition and machining processes on the same work station, a hybrid system is able to produce complicated geometry, especially the overhang structure with less or no support structures. Based on different geometry shapes, the five-axis system can save close to 
$100 \%$ of support materials. The surface quality of the final product is similar to the industrial milling capability. As there is a big demand in precision metal additive manufacturing applications, a more sophisticated process planner and general purpose for hybrid manufacturing systems is needed.

\section{ACKNOWLEDGMENTS}

This research was supported by the National Science Foundation Grant Numbers IIP-0637796, IIP0822739, and IIP-1345240, and the grant from the U.S. Air Force Research Laboratory contract \# FA8650-04-C-5704, and Missouri S\&T Intelligent Systems Center. Their support is greatly appreciated.

\section{REFERENCES}

[1] Han, L.; Liou, F.W.; and Phatak, K.M., (2005) "Modeling of Laser Cladding with Powder Injection," Metallurgical and Materials Transactions B, Volume 35B, pp. 1139-1150.

[2] J. Hur, K. Lee, h. Zhu, J. Kim, Hybrid rapid prototyping system using machining and deposition, Computer-Aided Design, 34 (2002) 741-754.

[3] Hur, Junghoon, Kunwoo Lee, and Jongwon Kim. "Hybrid rapid prototyping system using machining and deposition." Computer-Aided Design 34.10 (2002): 741-754.

[4] J. Gao, J. Folkes, O. Yilmaz, N. Gindy, Investigation of a 3D non-contact measurement based blade repair integration system, Aircraft Engineering and Aerospace Technology, 77 (2005) 34-41.

[5] Liou, Frank; Slattery, Kevin; Kinsella, Mary; Newkirk, Joseph; Chou, Hsin-Nan; Landers, Robert, "Applications of a Hybrid Manufacturing Process for Fabrication and Repair of Metallic Structures," Rapid Prototyping Journal, 2007, ISSN: 1355-2546, 2007 Volume: 13 Issue: 4 Page: 236 - 244.

[6] Ruan, J.; Eiamsa-ard, K.; and Liou, F., (2005) "Automatic Process Planning and Toolpath Generation of a Multi-Axis Hybrid Manufacturing System," SME Journal of Manufacturing Processes, Vol 7, No. 1, pp. 57-68.

[7] Liou, Frank; Slattery, Kevin; Kinsella, Mary; Newkirk, Joseph; Chou, Hsin-Nan; Landers, Robert, "Applications of a Hybrid Manufacturing Process for Fabrication and Repair of Metallic Structures," Rapid Prototyping Journal, 2007, ISSN: 1355-2546, 2007 Volume: 13 Issue: 4 Page: 236 - 244. 